\title{
Some effects of support on perceived shape of depicted objects
}

\author{
DARRELL L. BUTLER \\ Ball State University, Muncie, Indiana
}

\begin{abstract}
The purpose of this study was to provide a preliminary investigation on the effects of extraobject context on the perceived shape of depicted objects. Two hypotheses were examined: (1) support structures can affect apparent shape, and (2) parallel lines between objects affect apparent shape. Subjects made judgments about angles in depicted parallelopipeds. The parallelopipeds were depicted alone, supported by compression, and supported by tension. Some of the depictions contained parallel lines between support structures and objects. Results indicate context effects for objects supported by compression (all containing parallel lines between objects), but not for objects supported by tension. Parallel lines between support structure and object cannot account for the results of objects supported by tension.
\end{abstract}

Overall context and organization of pictures can affect the detection of objects in a single glance (Biedermann, $1972,1977,1981$ ) and the recognition of objects (Palmer, 1975). Based on his findings, Biedermann suggested that there are five types of picture contexts or picture constraints for each object: support, interposition, probability, position, and size.

Perkins and Cooper (1980) suggested that one of these extraobject picture constraints, support, may also affect perceived shape. Barrow and Tenenbaum (1981) provided an example that, in principle, supports this hypothesis. A variation of their example is provided in Figure 1. In this example, more observers reported that the small block is symmetrical in Figure 1A than in Figure 1B. Barrow and Tenenbaum argued that the influence of the near by coplanar surfaces on the perceived shape depends on connection through an implied ground plane that supports both objects.

An alternative hypothesis to explain Barrow and Tenenbaum's (1981) example is that parallel lines in the drawings affect perceived shape. Note that Figures 1A and 1B differ primarily in which lines are parallel. Recently, Butler and Kring (1987) showed that parallel lines in a depiction of an object can affect perceived shape, even when the parallel lines are widely separated; however, Butler and Kring's research did not consider whether parallel lines indicated support of the object.

One technique for discriminating these two hypotheses is to view Figure 1 upside down or in other orientations. If the perceived shape depends on an implied supporting ground plane, then perception should change as support

The author is indebted to Kimberly Baker, who ran most of the subjects in this experiment and in pilot work. Also, thanks are due to Sonja Binder and Patty Sawyer for helping to run subjects. This research was supported in part by a grant from the Ball State University Office of Research. Requests for reprints should be sent to the author at the Department of Psychological Sciences, Ball State University, Muncie, IN 47306. appears to change with changes in orientation. Alternatively, if the perceived shape depends on parallel lines, perception of shape should be the same when the object is viewed in any orientation.

Perception of shape does vary with orientation, thus implying that support is a more likely explanation than parallel lines for the context effect. Unfortunately, several alternative hypotheses confuse this conclusion. First, the change in orientation may affect the frame of reference. Rock (1983) argued that the frame of reference can exert a strong influence on information processing. Second, Rock's simple experiment (and previous research on support) does not discriminate between two types of support: compression and tension. An object sitting on a table is supported by compression. Compression is the type of support implied by Figure 1 when viewed upright. Suspended objects are supported by tension. When Figure 1 is viewed upside down, tension support is implied. Thus the changes in perceived shape seen in Figure 1 as it is reoriented could be due to changes in the type of support perceived.

In the present study, I took another approach to investigate the importance of support and parallelism on perception of object shape in depictions. In pilot studies, I was unable to produce reliable judgments that a depiction contained compression support without using parallel lines. However, depictions implying tension support do
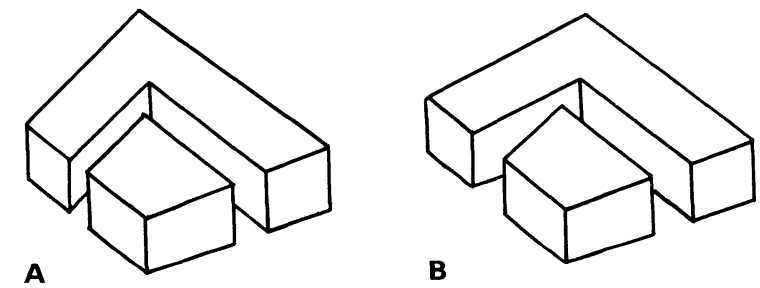

Figure 1. Illustration of extraobject context effects on perception (a variation of an example from Barrow \& Tenenbaum, 1981). 
not require parallel lines. This difference permits one to separate, at least in a general way, the effect of parallelism and support. In this experiment, subjects were asked to make angle judgments of depicted parallelopipeds presented alone, in a compression context, and in a tension context. Some of the tension contexts included parallel lines between support structure and object, and some did not.

\section{METHOD}

\section{Subjects}

There were 20 subjects, volunteers from undergraduate psychology courses. No students with uncorrected visual impairments were permitted to participate.

\section{Stimuli}

The stimuli were 12 parallelopipeds drawn on a black-and-white computer monitor. The parallelopipeds were created by first taking a sample of possible three-line intersections formed from two angles. Beginning at the three o'clock position, one angle (A) varied from $15^{\circ}$ to $105^{\circ}$ in $30^{\circ}$ intervals, and the second angle (B) varied from $15^{\circ}$ to $165^{\circ}$ in $30^{\circ}$ intervals. From the three-line intersections created by completely crossing these angles, all reflections and rotations were deleted, as done by Shepard (1981). Also all tee intersections (i.e., those in which the two smallest angles summed to $180^{\circ}$ ) were eliminated. Two different constructions were used to create parallelopipeds from the three-line intersections. If the intersection was an arrow (i.e., the two smallest angles summed to less than $180^{\circ}$ ), the intersection became the bottom left corner of a parallelopiped. If the intersection was a fork (i.e., the two smallest angles summed to more than $180^{\circ}$ ), the intersection became the center corner of a parallelopiped. All lines defining the parallelopipeds were the same length $(27 \mathrm{~mm})$ and width $(1 \mathrm{~mm})$. The tension context was created by drawing three vertical lines from the three top corners of the parallelopiped to the top of the display area on the CRT. The compression context was created by drawing three lines parallel to edges of the parallelopiped (implying a table top) and two vertical lines (implying table legs). Figure 2 shows examples of the tension and compression contexts. Pretests indicated that subjects perceived solid forms and appropriate support in these depictions. As can be seen in Figure 2, the angle to be judged was drawn in solid lines, and the rest of the drawing was composed of dotted lines. Dots were equally spaced, representing $20 \%$ of a solid line.

\section{Procedure}

The subjects were run one at a time. Instructions were presented by videotape. The subject sat in the testing cubical with the experiment CRT to the front and the video CRT to the right side. On a small table in front of the subject were two buttons and a dial that were connected to a computer. The subject was told that he/she would be judging the size of an angle in the surface of a depicted object, not simply the size of an angle as it appeared on a CRT. On each trial, one of the stimuli was presented in the upper left corner of the CRT and two other lines were presented in the lower right corner. These other two lines were the same length as the lines in the stimuli. The dial allowed the subject to continuously vary the angle between the two lines in the lower right between $0^{\circ}$ and $180^{\circ}$. One line remained in the three o'clock position, and the other line could be moved counterclockwise to $180^{\circ}$. Pressing one of the buttons ended the stimulus display. To return to the display, the subject pressed the same button. To end a trial, the subject pressed the other button, after a minimum 500-msec delay. Pilot studies showed that this two-button system lowered the probability that accidental buttonpresses would be recorded as responses. After the instructions were presented, stimuli were presented in three blocks: object alone, object supported by compression, and object supported by tension. Each block contained 22 stimuli, the 11 parallelopipeds crossed with the angle to
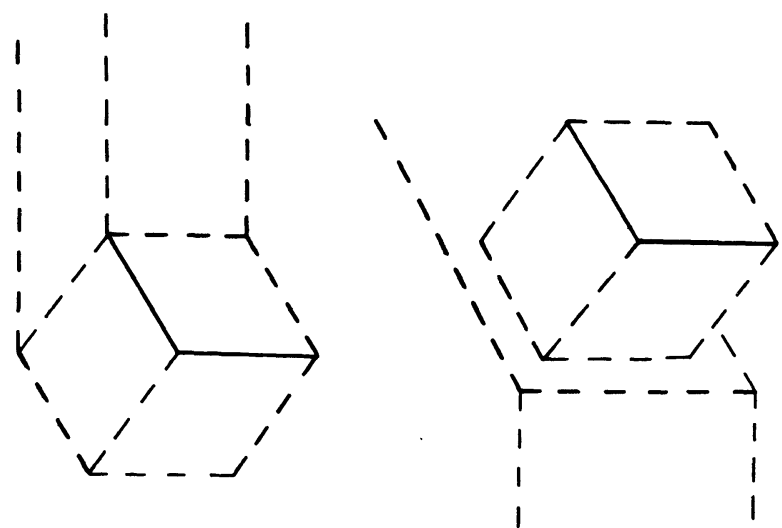

Figure 2. Examples of the types of stimuli used.

be judged (A or B). Order of blocks and order of stimuli within blocks were randomized independently for each subject.

\section{RESULTS}

The mean judgment time was $8.3 \mathrm{sec}$ per stimulus. Context had a significant effect on judgments. Judgments of each angle size were averaged across the other angle in the three-line intersection. An analysis of variance (ANOVA) was computed, using size of angle judged $\left(15^{\circ}\right.$ to $135^{\circ}$ ) and scene context (alone, supported by tension, and supported by compression) as independent variables. As expected, size of angle was a significant source of variance $[F(4,76)=58.61, p<.001]$. Context was also a significant source of variance $[F(2,38)=3.26, p<.05]$. The mean judgment of the stimuli in the compression context (86.6) was somewhat less than the mean judgments of the stimuli in the tension context (87.7) and the alone context (87.4). The interaction between angle and context was not significant $[F(8,152)=1.72$, n.s.], but separate analyses on each angle (presented below) indicate that context had a substantial effect on some parallelopipeds and not on others.

Separate ANOVAs run on each angle size, using the other defining angle in the intersection and scene context as independent variables, indicate effects of scene context. Context was a significant source of variance for $45^{\circ}$ angles $[F(2,38)=6.78, p<.01]$ and $75^{\circ}$ angles $[F(2,38)$ $=3.49, p<.05]$ and approached significance for $15^{\circ}$ angles $[F(2,38)=2.55, p<.08]$, but was not a significant source of variance for the $105^{\circ}$ angles $[F(2,38)=$ 1.77 , n.s.] or the $135^{\circ}$ angles $[F(2,38)=.46$, n.s. $]$. In general, these significant effects were due to the fact that the mean of the compression context was lower than the means of the other context conditions. Importantly, for the $45^{\circ}$ angle there was also a significant interaction between context and size of the other angle in the stimulus $[F(8,152)=2.85, p<.01]$. The interaction was produced primarily by judgments of the $45^{\circ}-45^{\circ}$ intersection. The mean in the compression context (81) was 
very small compared with that in the tension context (89) and the alone context (89).

\section{DISCUSSION}

Perceived shape does not appear to be a simple function of parallel lines. The compression contexts all were created using lines parallel to the parallelopiped. Means for these stimuli were somewhat smaller than those for equivalent stimuli in the other contexts. Four judgments were made of parallelopipeds in a tension context in which the lines indicating a support structure were parallel to lines in the parallelopiped. Compared with judgments of equivalent stimuli in other contexts, for one of these stimuli the mean judgment was the largest, for one it was the smallest, and for two it was virtually identical to the alone context. These results suggest that perceived shape may not depend on parallelism, at least in objects supported by tension.

The results of this experiment suggest that extraobject context affects shape perception. Perkins and Cooper (1980) suggested that perceived support may be an important factor. An alternative considered here was that parallel lines are an important factor. The present research suggests that compression support can affect perceived shape, but tension support cannot. All compression support drawings contained parallel lines, and it seems reasonable that the parallel lines would imply coplanarity and support. However, parallel lines in the tension context did not have a reliable effect. Thus, these results suggest that it is perceived compression support, not parallelism per se, that produces the effect.

\section{REFERENCES}

Barrow, H. G., \& Tenenbaum, J. M. (1981). Interpreting line drawings as three-dimensional surfaces. Artificial Intelligence, 17, 75-116. BiedermanN, I. (1972). Perceiving real world scenes. Science, 117, 77-80.

BIEDERMANN, I. (1977). On processing information from a glance at a single scene: Some implications for a syntax and semantics of visual processing. In S. Treu (Ed.), User-oriented design of interactive graphics systems. New York: ACM.

BiEDERMANN, I. (1981). On the semantics of a glance at a scene. In M. Kubovy \& J. R. Pomerantz (Eds.), Perceptual organization. Hillsdale, NJ: Erlbaum.

BUTLER, D. L., \& KRING, A. M. (1987). Integration of features in depictions as a function of size. Perception \& Psychophysics, 41, 159-164.

Palmer, S. E. (1975). The effects of contextual scenes on the identification of objects. Memory \& Cognition, 3, 519-526.

Perkins, D. N., \& CoOper, R. G., JR. (1980). How the eye makes up what the light leaves out. In M. A. Hagan (Ed.), The perception of pictures (Vol. 2, pp. 95-130). New York: Academic Press.

RocK, I. (1983). The logic of perception. Cambridge, MA: MIT Press.

SHEPARD, R. N. (1981). Psychophysical complementarity. In M. Kubovy \& J. R. Pomerantz (Eds.), Perceptual organization. Hillsdale, NJ: Erlbaum.

(Manuscript received for publication October 5, 1987.) 